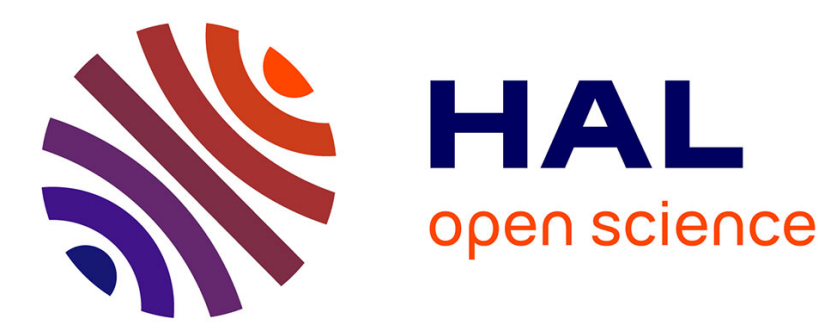

\title{
The homeomorphism of Minkowski space and the separable complex Hilbert space: the physical, mathematical and philosophical interpretations
}

\author{
Vasil Penchev
}

\section{To cite this version:}

Vasil Penchev. The homeomorphism of Minkowski space and the separable complex Hilbert space: the physical, mathematical and philosophical interpretations. 2021. hal-03437601

\author{
HAL Id: hal-03437601 \\ https://hal.science/hal-03437601
}

Preprint submitted on 20 Nov 2021

HAL is a multi-disciplinary open access archive for the deposit and dissemination of scientific research documents, whether they are published or not. The documents may come from teaching and research institutions in France or abroad, or from public or private research centers.
L'archive ouverte pluridisciplinaire HAL, est destinée au dépôt et à la diffusion de documents scientifiques de niveau recherche, publiés ou non, émanant des établissements d'enseignement et de recherche français ou étrangers, des laboratoires publics ou privés. 


\title{
The homeomorphism of Minkowski space and the separable complex Hilbert space: the physical, mathematical and philosophical interpretations
}

\author{
Vasil Penchev, vasildinev@gmail.com \\ Bulgarian Academy of Sciences: Institute of Philosophy and Sociology \\ Dept. of Logical Systems and Models
}

\begin{abstract}
A homeomorphism is built between the separable complex Hilbert space (quantum mechanics) and Minkowski space (special relativity) by meditation of quantum information (i.e. qubit by qubit). That homeomorphism can be interpreted physically as the invariance to a reference frame within a system and its unambiguous counterpart out of the system. The same idea can be applied to Poincaré's conjecture (proved by G. Perelman) hinting at another way for proving it, more concise and meaningful physically. Furthermore, the conjecture can be generalized and interpreted in relation to the pseudo-Riemannian space of general relativity therefore allowing for both mathematical and philosophical interpretations of the force of gravitation due to the mismatch of choice and ordering and resulting into the "curving of information" (e.g. entanglement). Mathematically, that homeomorphism means the invariance to choice, the axiom of choice, well-ordering, and well-ordering "theorem" (or "principle") and can be defined generally as "information invariance". Philosophically, the same homeomorphism implies transcendentalism once the philosophical category of the totality is defined formally. The fundamental concepts of "choice", "ordering" and "information" unify physics, mathematics, and philosophy and should be related to their shared foundations.

Keywords: axiom of choice, choice, general relativity, gravitation, Hilbert space, information, Minkowski space, ordering, Poincaré's conjecture, pseudo-Riemannian space, quantum information, qubit, well-ordering
\end{abstract}

\section{INTRODUCTION: THE UNIFICATION OF "VECTOR" AND "FUNCTION" IN HILBERT SPACE AND ITS MEANING FOR QUANTUM MECHANICS}

The set of all complex numbers, $\boldsymbol{C}$ is granted. Then the corresponding set of all subsets of $\boldsymbol{C}$ is the separable complex Hilbert space $\mathscr{H}$.

There is one common and often met identification of $\mathscr{H}$ with the set $\mathbb{H}$ of all ordinals of $\mathscr{H}$, which rests on the identification of any set with its ordinal. However, if any ordinal is identified as a certain natural number, and all natural numbers in Peano arithmetic are finite ${ }^{1}$, $\mathscr{H}$ and $\mathbb{H}$ should not be equated, for $\mathscr{H}$ includes actually infinite subsets of $2^{C}$. Here "actually infinite subset" means 'set infinite in the sense of set theory'.

Furthermore, $\mathcal{H}$ is identified as the set $\mathbf{H}$ of all well-ordered sets, the elements of which are elements of some set of $2^{C}$, i.e. in other words, the elements of $2^{C}$ considered as classes of equivalency by ordering are distinguished by ordering within any class of that ordering.

Those distinctions can be illustrated by the two basic interpretations of $\mathscr{H}:(1)$ as the vectors of n-dimensional complex generalization of the usual 3D real Euclidean space, isomorphic to $\mathbf{H}$;

\footnotetext{
${ }^{1}$ This is a property implied by the axiom of induction.
} 
and (2) as the square integrable functions, isomorphic to H. The latter adds to the former unitarity (unitary invariance), which is usually interpreted as energy conservation in their application in quantum mechanics. By reflection back, energy conservation is a physical equivalent of both (3) equivalence after ordering and (4) actual infinity, i.e. to (5) the concept of ordinal number in set theory.

On the contrary, once one does not involves energy conservation, e.g. generalizing it to energy-momentum conservation as in the theory of general relativity or that of entanglement, $\mathbf{H}$ rather than $\mathscr{H}$ is what should be used unlike quantum mechanics based on $\mathscr{H}$, and actual infinity avoided or at least precisely thought before utilizing.

Furthermore, (6) the relation between $\mathscr{H}$ and $\boldsymbol{H}$ can be interpreted as the 3D Euclidean space under (7) the additional condition of cyclicality (reversibility) of $\boldsymbol{H}$ conventionally identifying the first "infinite" element with the "first" element of any (trans)finite well-ordering. Indeed, the axiom of induction in Peano arithmetic does not admit infinite natural numbers ${ }^{2}$. If one needs to reconcile both finite and transfinite induction to each other, the above condition is sufficient.

It should be chosen for Poincaré (1904)'s conjecture proved by G. Perelman (2002; 2003). If that condition misses, the topological structure is equivalent to any of both almost disjunctive domains $^{3}$ of Minkowski's space of special relativity ${ }^{4}$ rather than to a 4D Euclidean ball. The two domains of Minkowski space $M$ can be interpreted as two opposite, "causal directions" resulting in both reversibility of the 3D Euclidean space and topological structure of the above 4D ball.

The relation between $\mathscr{H}$ and $\boldsymbol{H}$ generates any of the two areas of $\mathcal{M}$ as follows. Both unitarity of $\mathscr{H}$ and non-unitarity of $\boldsymbol{H}$ for any ordinal $n$ and any well-ordering of length $n$ are isomorphic to a 3D Euclidean sphere ${ }^{5}$ with the radius $r(n)$. All those spheres represent the area at issue.

That construction can be interpreted physically as well. Energy (E) conservation as unitarity represents the class of equivalence of any ordinal $\mathrm{nn}$. If the concept of physical force (F) is introduced as any reordering, i.e. the relation between any two elements of the above class, it can be reconciled with energy conservation (unitarity) by the quantity of distance $(x)$ in units of elementary permutations for the reordering so that $F . x=E$.

Back seen, both (6) and (7) imply Poincaré's conjecture and thus offer another way of its proof.

One can discuss the case where $\mathscr{H}$ is identified with $\boldsymbol{H}$ and what it implies. Then (8) the axiom of induction in Peano arithmetic should be replaced by transfinite induction correspondingly to (4) above, and (9) the statistical ensemble of well-orderings (as after measurement in quantum mechanics) should be equated to the set of the same elements (as the coherent state before measurement in quantum mechanics) for (3) above.

\footnotetext{
${ }^{2}$ The natural number " 1 " is finite. The successor of any finite natural number is finite. Consequently, all natural numbers are finite for the axiom of induction.

${ }^{3}$ They are almost disjunctive as they share the light cone.

${ }^{4}$ Indeed, special relativity is a causal theory, which excludes the reverse causality implied by cyclicality.

${ }^{5}$ This means the surface of a 3D Euclidean ball (an electronic copy is available at:

https://ssrn.com/abstract=3632159 ).
} 
In fact, that is the real case in quantum mechanics for unitarity as energy conservation is presupposed. Then (8) implies the theorems of absence of hidden variables in quantum mechanics (Neumann 1932; Kochen. Specker 1968), i.e. a kind of mathematical completeness interpretable as the completeness of quantum mechanics versus Einstein, Podolsky, and Rosen (1935)'s hypothesis stating the incompleteness of quantum mechanics:

The (8) and (9) together imply the axiom of choice. Indeed, the coherent state (the unordered set of elements) excludes any well-ordering for the impossibility of hidden variables implied by (8). However, it can be anyway well-ordered for (9). This forces the well-ordering principle ("theorem") to be involved, which in turn is equivalent to the axiom of choice.

Furthermore, $H_{\mathcal{H}}$ can be represented as all sets of qubits.

A qubit is defined in quantum mechanics and information as the (10) normed superposition of two orthogonal ${ }^{6}$ subspaces of $\mathscr{H}$ :

$$
Q^{\stackrel{\text { def }}{=}} \alpha|0\rangle+\beta|1\rangle
$$

$|0\rangle,|1\rangle$ are the two orthogonal subspaces of $\mathscr{H}$; and $\alpha, \beta \in C:\left.|\dot{\alpha}+| \beta\right|^{2}=1$.

Then, (11) $\mathrm{Q}$ is isomorphic to a unit 3D Euclidean ball, in which two points in two orthogonal great circles are chosen so that the one of them is on the surface of the ball.

That interpretation is obvious mathematically. It makes sense physically and philosophically for the above consideration of space as the relation of $\mathcal{H}$ and $\boldsymbol{H}$.

Now, it can be slightly reformulated and reinterpreted as the joint representability of $\mathscr{H}$ and $\boldsymbol{H}$, and thus their unifiability in terms of quantum information.

Particularly, any theory of quantum information, including quantum mechanics as far as it is so representable, admits the coincidence of model and reality: right a fact implied by the impossibility of hidden variables in quantum mechanics for any hidden variable would mean a mismatch of model and reality.

$\boldsymbol{H}$ can be interpreted as an equivalent series of qubits for any two successive axes of $\boldsymbol{H}$ are two orthogonal subspaces of $\mathscr{H}$ :

$\left\{C_{j}\right\} \in \boldsymbol{H}$; then (12). any successive pair $\left(C_{j}, C_{j+1}\right)=Q_{j+1} ; Q_{j+1} \in \boldsymbol{Q}$ under the following conditions:

(13) $\alpha_{j+1}=\frac{C_{j}}{\sqrt{C_{j}^{2}+C_{j+1}^{2}}} ; \beta_{j+1}=\frac{C_{j+1}}{\sqrt{C_{j}^{2}+C_{j+1}^{2}}}$

(14) $\alpha_{1}=0 ; \beta=\frac{C_{1}}{\left|C_{1}\right|}$

(15) If both $C_{j} C_{j+1}=0$, then: $\alpha_{j+1}=0 ; \beta_{j+1}=1$.

(14) and (15) are conventional, chosen rather arbitrarily only to be conserved a one-to-one mapping between $\boldsymbol{H}$ and $\boldsymbol{Q}$.

\footnotetext{
${ }^{6}$ Any two disjunctive subspaces of $\mathscr{H}$ are orthogonal to each other.
} 
$Q$ is intendedly constructed to be ambivalent to unitarity for any qubit is internally unitary, but the series of those is not. Furthermore, one can define n-bit where a qubit is 2-bit therefore transforming unitarily any non-unitary n-series of complex numbers. The essence of that construction is the double conservation between the two pairs: "within - out of" and "unitarity non-unitarity".

That conservation is physical and informational, in fact. The simultaneous choice between many alternatives being unitary and thus physically interpretable is equated to a series of elementary or (at least more elementary) choices. Then, the "physical" inside will look like the "chemical" outside and vice versa". If a wholeness such as the universe is defined to contain internally its own externality, this can be modeled anyway consistently equating the non-unitary "chemical" and unitary "physical" representations in the framework of a relevant physical and informational conservation.

H can be furthermore interpreted as all possible pairs of characteristic functions of independent probability distributions and thus, of all changes of probability distributions of the state of a system, e.g. a quantum system.

Practically all probability distributions and their characteristic functions of the states of real systems are continuous and even smooth as usual. The neighboring values of probability implies the neighborhood of the states. Thus, the smoothness of probability distribution implies a well-ordering and by the meditation of it, a kind of causality: the probability of the current state cannot be changed jump-like.

This is an expression of a deep mathematical dependence (or invariance) of the continuous (smooth) and discrete. The probability distribution can mediate between them as follows:

H can be defined as the sets of the ordinals of $\boldsymbol{H}$ where a representative among any subset of the permutations (well-orderings) of $n$ elements is chosen according a certain and constructive rule. That rule in the case in question is to choose that permutation (well-ordering), the probability distribution of which is smooth.

Particularly, the homotopy of $\boldsymbol{H}$ can identified with, and thus defined as that mapping of $\boldsymbol{H}$ into $\mathscr{H}$ conserving the number of elements, i.e. the dimensionality $n$ of the vector between $\boldsymbol{H}$ and H. If $\boldsymbol{H}$ is interpreted as the "set of types" on $\boldsymbol{C}$, this implies both "axiom of univalence" [IAS 2013] and an (iso)morphism between the category of all categories and the pair of $\mathcal{H}$ and $\boldsymbol{H}$.

That consideration makes obvious the equivalence of the continuous (smooth) and discrete as one and the same well-ordering chosen as an ordinal number among all well-orderings (permutations) of the same elements and it by itself accordingly. In other words, the continuous (smooth) seems to be a class of equivalence of the elements of a set (including finite as a generalization of continuity as to finite sets).

\footnotetext{
${ }^{7}$ That statement can be made precocious and mathematically rigorous (Penchev 2019) and furthermore, it can be intuitively justified by the thought experiment in Einstein's manner suggested there: by the indistinguishability of the signal of a certain chemical substance arriving from any distant star due to "red shift" and the measurement of the same substance however simultaneously being relevantly entangled and situated in a laboratory on Planet Earth.
} 
Furthermore, the same consideration can ground (3) and (9) above, i.e. the way in which a coherent state before measurement is equivalent to the statistical ensemble of measured states in quantum mechanics. The same property can be called "invariance to choice" including the invariance to the axiom of choice particularly.

This means that the pure possibility, e.g. that of pure existence in mathematics, also interpretable as subjective probability should be equated to the objective probability of the corresponding statistical ensemble once unitarity (energy conservation) has already equated He and $\boldsymbol{H}$.

Indeed, the set or its ordinal number (i.e. the set of well-orderings) can be attributed to the elements of $\mathscr{H}$ and the statistical mix of all elements of $\boldsymbol{H}$ corresponding to a given element of $\mathscr{H}$. Any measurement ascribes randomly a certain element of the corresponding subset of $\boldsymbol{H}$ to any given element of $\mathscr{H}$. Thus, measurement is not unitary, e.g. as if it is due to a „collapse of wave function".

Then, $\mathscr{H}$ and $\boldsymbol{H}$ can be interpreted as two identical, but complementary dual spaces of the separable complex Hilbert space. Initarity means their identity, and the non-unitarity of measurement representing a random choice means their complementarity.

That "invariance to choice" can ground both so-called Born probabilistic (e.g. Born 1954) and Everett (\& Wheeler) "many-worlds" interpretations of quantum mechanics (Everett III 1957; DeWitt, Graham 1973; Wheeler, Zurek 1983). The former means the probability for a state to be measured or a "world" to take place, and the former complements that consideration by the fact that all elements constituting the statistical ensemble can be consistently accepted as actually existing.

One can emphasize that the Born interpretation ascribes a physical meaning of the one component (namely the square of the module as probability) of any element of the field of complex numbers underlying both $\mathscr{H}$ and $\boldsymbol{H}$. After that, the physical meaning of the other component, the phase is even much more interesting. It should correspond to initarity, and then, it seems to be redundant, i.e. the field of real numbers would be sufficient, on the one hand, but furthermore, to time, well-ordering, and choice implied by it.

In other words, just the phase is what is both physical and mathematical "carrier" and "atom" of the invariance of choice featuring the separable complex Hilbert space.

\section{POINCARÉ'S CONJECTURE AND ITS SIMPLE, “QUANTUM" PROOF IN VIRTUE OF THE HOMEOMORPHISM AT ISSUE}

If one interprets Poincaré's conjecture in terms of Minkowski space (in turn equivalent to the qubit Hilbert space in virtue of the homeomorphism at issue) one can reduce it therefore proving the hypothesis simply and immediately referring only to a few premises ${ }^{8}$ :

8 That homeomorphism is rather extraordinary as a homeomorphism because it means a smooth transformation of a continuous (even smooth) vector space (such as Minkowski space) into a vector space with an enumerable basis (such as the separable complex Hilbert space). This is possible only if the axiom of choice is involved and corresponds to the fundamental reason for the latter to be utilized in quantum mechanics. However, that kind of homeomorphism should not be surprising as far as topology suggests 
1. The light cone of Minkowski space (which is the same for both domains) is homeomorphic to 3D unfolding (i.e. in the usual Euclidean space) of the unit 3-sphere (which refers to the four-dimensional generalization of that Euclidean space ${ }^{9}$ ).

2. The light cone is isomorphic (and thus, trivially homeomorphic) to Euclidean space whether in virtue of special relativity as relevant theory referring to our experience and physical experiments in Euclidean space, or directly representing the light cone as the propagation of the spherical light wave passing successively and unambiguously all points of Euclidean space.

3. Applying (1) and (2) to Poncaré's conjecture, it is reduced to the statement (to be proved): the $3 \mathrm{D}$ unfolding of the unit 3 -sphere is homeomorphic to the 3 -sphere itself ${ }^{10}$. It can be verified immediately in virtue of the following propositions:

4. Minkowski space is homeomorphic (and thus, isomorphic) to the qubit Hilbert space (as it is demonstrated above).

5. The qubit Hilbert space just as the separable complex Hilbert space, from which it originates, is unitarian. That is: any ordering (e.g. "after measurement") of the qubits of a certain wave function is equivalent to its coherent state (e.g. "before measurement").

6. The homeomorphism meant in (4) transfers the unitarily of the qubit Hilbert space to Minkowsky space as well.

7. That unitarity of Minkowski space implies its homeomorphism to the unit 3-sphere which should be proved.

If (7) does not seem obvious enough, one can comment on it in detail. Minkowski space is fully ordered and even well-ordered in relation to a certain moment granted to be the present one, generating however, two well-orderings: the one is past, ending in the present moment, and the other one is future, starting from the present moment. Thus, the fourth, non-convex dimension of Minkowski space is granted to represent time modeling special relativity.

On the contrary, the unit 3-sphere is not fully ordered (or it is "two times" well-ordered) by itself. Anyway, one can complement it to both well-orderings merely and conventionally choosing a certain 2-sphere (i.e a usual 3D ball) as the beginning (respectively, the end) of the

set theory (and thus, the axiom of choice) as its ground. Furthermore, the same homeomorphism is called "isomorphism" in other papers by virtue of the following consideration. One can neglect that those vector spaces are topological spaces (supplied by quite natural topologies) if their metric properties are not meant as well. However, the present paper involves their topologies explicitly since Poincaré's conjecture (being topological properly) and its generalization in pseudo-Riemannian space is a main subject of investigation: according, that isomorphism should be discussed just as homeomorphism.

${ }^{9}$ An additional, the "fourth" dimension, in which the light cone is "folded" being the 3D unfolding of a 3 -sphere is meant. That additional link in the "fourth" dimension is a new topological property preventing the direct homeomorphism of the light cone into a 3-sphere: and thus, the usual Euclidean space into the unit 3-sphere (properly, Poincaré's conjecture).

${ }^{10}$ This seems to be a counterintuitive and even self-contradictory statement (but only at first glance) for a 3-dimensional figure (such as the light cone) needing to be homeomorphic to a 4-dimensional figure (such as the unit 3-sphere). In fact, the solution of the alleged paradox consists in the infinite "size" of the 3-dimensional figure being able to be transformed painlessly into the additional "fourth" dimension necessary for the finite ("unit") 4-fimensional figure. Indeed, infinity means not more than a second, thus additional dimension to finiteness. 
unit 3-sphere. Properly, the same unit ball can be granted as a cut of the 3-sphere after which it is able to be unfolded as Minkowski space (and after a few technical conditions being nonessential in the present, conceptual comment). Then, the unitarity of Minkowski space (inherited from the qubit Hilbert space) means the rather obvious identity of the unit 3-sphere whether a certain 3D ball is chosen or not.

That "quantum" pathway (except it is extraordinarily direct and short) from proving Poincaré's conjecture allows for additional reflections on the physical and mathematical sense and meaning of the separable complex Hilbert space of quantum mechanics involving the very powerful tool of unitarity. That tool manages to resolve a much wider area of similar problems than what is intended initially: to be unified the discrete (forced by the Planck constant) and smooth description of the apparatus by classical physics. For example, it explicates the physical sense and meaning of infinity as equivalent to an additional dimension, by which Poincaré's conjecture is quite directly provable and furthermore, revealing its physical and philosophical connotations.

\section{POINCARÉ'S CONJECTURE ABOUT THE PSEUDO-RIEMANNIAN SPACE OF GENERAL RELATIVITY: GRAVITATION}

What is up if one substitutes the "flat" Minkowski space of special relativity with the "curved" pseudo-Riemannian space of general relativity investigating again the above "quantum" proof of Poincaré's conjecture, first of of all due to the problematic unitarity of Einstein's gravitation resulting in the impossibility of "quantum gravity":

Poincaré's conjecture so generalized would question whether Euclidean space already supplied by gravitational field nonetheless is homeomorphic to the 3-sphere (as far as any homeomorphic deformation once the 3-sphere has been situated in pseudo-Riemannian space is homeomorphic to the 3 -sphere at issue, i.e. in the four-dimensional Euclidean space). A few preliminary notices elucidating the importance and meaning of that extended hypotheses are relevant:

The gravitation of general relativity does not seem to be unitarian for the standard understanding of unitarity needs the metric commutativity of pseudo-Riemannian space being obviously violated due to its curvature and appearing in particular in the generalized law of energy-momentum conservation rather than properly energy conservation shared by the "classical" quantum mechanics: a fundamental obstacle preventing many theories of quantum gravity.

Nowever, the above sketch of an eventual "quantum" proof of Poincaré's conjecture replaces the metric commutativity with a generalized, "topological" commutativity since the 2-spheres of light cone of Minkowski space are homeomorphic to the "axes" of the separable complex Hilbert space (respectively, to the qubits of the qubit Hilbert space): indeed, the are only "flatly" homeomorphic unlike the "curvely" homeomorphic 2-spheres of pseudo-Riemannian space, but this is not important since only the availability of unambiguous homeomorphism is essential. Then, that generalized "topological" commutativity is already able to include the energy-momentum conservation of general relativity in the framework of a relevantly 
generalized topological unitarity (for energy-momentum conservation is in the scope of the topological generalization of energy conservation).

One can further observe that the topological generalization is not so radical, in fact, as it seems at first glance for the following consideration. Classical mechanics divides disjunctively (as in a tensor product) the metrically constant (or invariant) space (this is Euclidean space, but this is not essential to the consideration) from any metrically arbitrary trajectory occurring within it. However, the class of all possible trajectories includes only those homeomorphic to each other. That essence of the description of classical mechanics remains the same in both quantum mechanics and general relativity; indeed:

Quantum mechanics only replaces Euclidean space with the separable complex Hilbert space, and "trajectory" with "wave function". However, as if general relativity does not share the same structure of description (but only seemingly) since both "space" and a "world line" within it are variable and arbitrarily "curve" in general: even quite not, for the world line can be granted to be "flat" as a geodesic line in an additionally curved space, as well as vice versa: the space can be postulated to be the Mikowski one, and the world line to be curved additionally and compensatorily (in order to include the curvature of the space once it is granted to be "flat" being Minkowski space).

Then, metric commutativity meant literally by the unitarity of the "classical" quantum mechanics refers only to the space whichever it be in the specific description of either classical mechanics or general relativity, or quantum mechanics. On the contrary, topological commutativity (as it is exemplified a few times above) is universal being shared as by both trajectory and space as by each of the three enumerated descriptions. Thus, it does not need the opposition of absolutely distinguished "space" (whichever it be in each case) and "trajectory" within it (and thus, corresponding to its nature in each case) since both are able to share topological commutativity. Particularly, one can speak of two or more interacting trajectories in classical physics, or of two or more interacting spaces in quantum information studying entanglement. Grantig the relevant topological unitarity, one can choose the simplest description to study any subclass of phenomena in the framework of the general class sharing the topological unitarity. As far as topological unitarity is equivalent to quantum-information conservation, the general class at issue can be specified by that conservation.

Then, one can conclude that the generalized hypothesis of Poincaré (as it is formulated above in this section) can be proved in virtue of the same quantum proof as the original one: and the generalized conjecture implies the very important corollary about the topological unitarity of Einstein's gravitation shared by the unit 3-sphere in turn sharing the SU(2) symmetry of weak interaction even metrically. So one can utilize the method of "Wittgenstein's ladder" (as what topological unitarity is) to pioneer also the metric unitarity of general relativity turning out to be isomorphic to that to which weak interaction can be defined: $\mathrm{SU}(2)$.

Both the gravitation of general relativity and the weak interaction of the Standard model are violations of SU(2); but the Higgs mechanism able to generate mass at rest by the Higgs field (respectively, the Higgs boson) just as a "spontaneous violation of symmetry SU(2)" is very well 
studied already. One can suggest that mass at rest in virtue of the Higgs mechanism in the Standard model and the mass-energy of general relativity violating the same SU(2) symmetry (being the symmetry of the 3-sphere) can and should be described uniformly (after which Einstein's gravity can be interpreted as quantum gravity not worse).

One can complement the consideration about the rest two symmetries of the Standard model, $\mathrm{U}(1)$ and SU(3) and their relation to the 3-sphere and Poincaré's conjecture using SU(2) as a bridge between the former and the latter meaning the unification of $U(1)$ and $S U(2)$ in electroweak interaction as well as the doubling of electroweak interaction into SU(3) (strong interaction) in relation to momentum, accordingly.

As far as $U(1)$ can be visualized as the symmetry of a 2-sphere, and SU(2), as that of a 3 -sphere, the unification of electroweak interaction means that 2-sphere to belong to the 3-sphere and thus, to be the class of equivalence of 2 -spheres sharing the same set of a certain unfolding of the 3-sphere in the four dimension (which is real in the 4-dimensional Euclidean space, but its counterpart in Minkowski space is imaginary and physically interpretable as time in special relativity). Then, the class of equivalence of $U(1)$ in relation to all $U(1)$ to which $S U(2)$ is decomposable can be interpreted physically as all elements sharing the same world line (or in other words, the same "course of time") and projectable into a certain light 2 -sphere (i.e. belonging to the light cone). That consideration is reasonable to be restricted only to the reversible time of a coherent quantum state (only in the interval of which to be interpreted the "same course of time" unifiable into the class of equivalence of SU(2) as U(1)).

The quark model of strong interaction means an absolute analogy of SU(3) to the unified symmetry of electroweak interaction however without the option of decomposability to two separate symmetries (which corresponds to the hypothetical confinement prohibiting the existence of free quarks), but including the same Higgs mechanism for generating mass at rest by the spontaneous violation of symmetry. Nowever, one admits an analogue of U(1) in SU(2) to inseparable symmetry of SU(3), its sense of a class of equivalence to the alleged analogue of $\mathrm{SU}(2)$ into the framework of SU(3) would correspond physically to the integral of force during the course of (reversible) time.

The 3-sphere sharing SU(2) and relevant to the eventual "topological unitarity" of Einstein's gravitation, and thus, to the suggestable correspondence of the Higgs mechanism (available in both cases of electroweak and strong interaction) and mass-energy in general relativity is able to link essentially gravitational interaction to those of the Standard model. Then the 3-sphere conserves the topological properties of Euclidean space for their homeomorphism in virtue of Poincaré's conjecture doubtlessly proved by G. Perelman (and recognized by Clay Mathematics Institute as the solution of a problem among the seven problems of Millennium, heralded by it).

The present paper insists on the physical interpretation and fundamental sense of Poincaré's conjecture, furthermore suggesting the option for another, "quantum" proof of the same statement. The physical importance of the homeomorphism of Euclidean space and the unit 3-sphere consists in the proof of the way in which the smoothness of classical mechanics (shared bt general relativity and by the description of the apparatus in quantum mechanics) can be 
transferred logically and mathematically consistently to the fundamental symmetries of the Standard model and thus, to quantum mechanics inherently discrete for the Planck constant.

\section{CHOICE AND ORDERING IN PHYSICS: QUANTUM INFORMATION}

Unitarity, furthermore generalized as topological unitarity (as above), can be considered also abstractly as a relation of choice and ordering, therefore postulated in the foundation of mathematics as the axiom of choice in set theory (and equivalent to the well-ordering theorem): thus, pioneering its formulation as a philosophical concept as well.

The notion of unitarity originates from quantum mechanics by the unification of Heisenberg's matrix mechanics and Schrödinger's ondulatory mechanics and their reconciliation in the separable complex Hilbert space being unitary in definition (and even chosen just for its unitarity). However, the unitarity exemplified for the specific problem and objective of quantum mechanics by that Hilbert space is rooted in the foundations of mathematics (by the axiom of choice) and can be reduced to that basis independently of all additional (being many, but accidental) properties in quantum mechanics.

Then, its essence can be revealed just in the relation of "choice" and "ordering" postulated to be equivalent by the axiom of choice and the well-ordering theorem even for infinite sets only continuing the trend of the same property of all finite sets not needing it or any other additional axiom to be proved.

The concept of algorithm is what is able to relate "choice" and "ordering" as to all finite sets: a property, which allows for it to relate computer science and mathematics directly. In fact, it excludes any fundamental randomness "by itself" for the existence of a relevant algorithm (and an ordering in the final analysis) though eventually unknown allowing for any element of any finite set to be chosen unambiguously.

Thus, the concept of unitarity can be considered as a counterpart of "algorithm" also relevantly generalized as to infinite sets: they might be equated to mean the same, at least as to the relation of "choice" and "ordering" and opposed only by "infinite set versus finite set" correspondingly. Indeed, the unitarity of the separable complex Hilbert space and exploited by quantum mechanics unprecedentedly involves the axiom of choice as a necessary condition of an experimental science (what quantum mechanics is) ${ }^{11}$ and it (unitarity) can be granted to be an algorithm at all, infinite and fundamentally unknown, allowing the states before and after measurement to be equated always, therefore allowing for quantum mechanics to be a natural science.

That consideration is called to elucidate which is to be the "algorithmic counterpart of topological unitarity" as it is introduced above due to the eventual "quantum" proof of Poincaré's

\footnotetext{
${ }^{11}$ Here is how, explicitly and in detail. The coherent quantum state before measurement cannot be ordered fundamentally just in virtue of the theorems of the absence of hidden variables in quantum mechanics (Neumann 1932; Kochen, Specker 1968). On the contrary, the sets of all results (i.e. after measurement) are well-ordered necessarily for the parameter of the time of registration (or other similar ones). Then, if both are equated (necessary for quantum mechanics to be an objective science), this implies the well-ordering "theorem" and thus, the axiom of choice.
} 
conjecture. The usual unitarity (i.e. which is not "topological") means that all elements of the infinite set at issue are equally probable and choosable; respectively all branches of algorithms are not specified additionally by any probability to happen because all of them do not share any nonzero intersections between each other ${ }^{12}$.

On the contrary, topological unitarity allows for those elements to be individually specified by their unique probabilities (different in general); or respectively, any (though, with the same and equal probability) element to possess many identical copies therefore increasing the chance of being chosen. This makes sense only if the corresponding algorithms are probabilistic, which means: (1) at least two branches share nonzero intersection; (2) the corresponding probabilities manage which branch to be chosen after a statistical ensemble of branches between those branches.

Consequently, the algorithmic counterpart of topological unitarity is probabilistic necessarily, and as far as all quantum algorithms can be identified as all probabilistic ones, the counterpart in question is to be "quantum algorithm".

Furthermore, the correspondence of "choice" and "ordering" can be described unambiguously by a certain wave function (also being isomorphic to a characteristic function of some probability distribution) in each case. This can be visualized by the following consideration:

The axiom of choice guarantees that any element of any infinite set (in the scope in which the axiom is granted to be valid) can be chosen, Nonetheless, it states noting about the probability of that element to be chosen in a statistical ensemble of choices of all elements of the set at issue therefore admitting any probability density distribution about that ensemble only under the condition the value of probability not to be zero for any element. Furthermore, the axiom of choice states nothing about whether the chosen element has to be returned after choice or not thus generating different statistical ensembles in the former versus the latter case. Anyway, if one researches the correspondence of the axiom of choice and the well-ordering "theorem", the latter case of non-returning seems to be the natural suggestion.

Then considering a statistical ensemble of orderings of the same infinite set in virtue of the axiom of choice and its correspondence to the well-ordering theorem, the elements will be ordered with great probability according to their probability to be chosen and described by that probability density distribution. Thus, though all orderings are theoretically possible, only a finite set of orderings would be observed after a finite set of choices. Meaning that observation, the following conventional definition about the bijection of an infinite set into a finite set ${ }^{13}$ can be granted under complementing by the corresponding probability density distribution (respectively, by its characteristic function being furthermore isomorphic to a certain wave function): that bijection is described unambiguously by the corresponding probability density distribution (wave function).

\footnotetext{
${ }^{12}$ Indeed, any nonzero intersection of algorithmic branches admits a probabilistic choice between them.

${ }^{13}$ Furthermore, it can be grounded absolutely rigorously if one takes in account the Skolem (1922) relativity of the notion of set and the Dedekind (1888) set-theoretic definition of finitiness as this is cleared up in another paper (Penchev 2020 August 25).
} 
In turn, the same definition allows for the following, purely set-theoretical definition of "wave function" (or respectively, that of the quantum state determined by it) as the bijection of an infinite set (which can be philosophically reflected as the state "by itself", or before measurement) into a finite set (accordingly, the same state, but "for us", or after measurement).

Then if unitarity at all means the axiom of choice in the case of equiprobable choice of any element belonging to the set, topological unitarity refers to the general case where the choice is additionally determined by a relevant probability density distribution for any element of the set to be chosen. The latter case of topological unitarity admits furthermore quantum algorithms able to process unambiguously probability density distributions (now interpreted as relevant to the probability of any element of an infinite set to be chosen in virtue of the axiom of choice and its correspondence to the well-ordering "theorem").

The same observation implies that "quantum algorithm" can be defined equivalently both probabilistically (i.e. in relation to the elements of the infinite set) and deterministically (i.e. in relation to the probabilistic density distributions featuring any of those sets as a whole). The latter case means a standard Turing machine however processing qubits (i.e. probability density distributions e.g. in virtue of topological unitarity) rather than bits (Penchev 2020 July 22). Particularly, and practically this means that the general model of quantum computer can be essentially simplified considering it as a Turing machine processing a quantum tape consisting of qubits rather than of bits (Penchev 2020 August 5).

Finally, what topological unitarity means after it refers to qubits should be the quantity measured in qubits, i.e. quantum information, to which a generalized conservation is to be related, namely, the conservation of quantum information (Penchev 2020 October 5) just as the usual unitaririty of the "classical' quantum mechanics relates to energy conservation. Obviously, the former case is the general one as to unitarity as to conservation.

\section{CHOICE AND ORDERING IN MATHEMATICS: INFORMATION}

The consideration in the last paragraph though meaning the relation of "choice" and "ordering" in physics is abstract enough (presentable even only in set-theoretical terms and concepts), so it can be transferred directly into the foundations of mathematics researching for initarity (including topological intarity) and involving "Hilbert arithmetic" (Penchev 2021 August 24) as the relevant tool for investigating the shared, "Pythagorean" basis of mathematics (arithmetic, logic and set theory) and quantum mechanics (the separable complex, or qubit, Hilbert space).

Thus, the objective now is how "topological unitaririty" (and the "classical" unitarity before that) to be formulated only in the framework of Hilbert arithmetic in a way which would allows for information (whether quantum or classical) to be defined maximally wide and related to the foundations of mathematics (more exactly: propositional logic, the axioms of Peano arithmetic, and set theory). That representation of (topological) unitarity and (quantum-information) conservation in the framework of Hilbert arithmetic is so simple that it challenges the usual scientific and mathematical "common sense" used to like the Cartesian abyss between physics and mathematics and their resultative complicatedness and sophistication: 
So, topological unitarity means the relation of "ordering" and "choice" in the dual Peano arithmetic (i.e. the initial Peano arithmetic serves as a "reference frame" ${ }^{\text {", }}$, in which an "observer"14 is situated) unlike unitarity in a narrow sense, referring to the proper Peano arithmetic and thus relevant to "Gödel mathematics"15 (what contemporary mathematics is, and its philosophy means as "mathematics" at all, i.e. ostensibly: without alternatives): in other words, it is not related to Hilbert arithmetic or to the dual Peano arithmetic therefore identifying naively the initial Peano arithmetic as the proper one.

That definition implies a few corollaries with very essential interpretations: (1) the idempotence of "unitarity" and "topological invariance"; (2) the idempotence of "energy conservation" and "quantum-information conservation"; (3) the relativity of "energy conservation" and "quantum-information conservation" furthermore linked to Skolem (1922)'s "relativity of the notion of set"; as well as a meta-observation about (4) the conceptual neighbourhood of "idempotence" and "relativity":

(1) About the idempotence of "unitarity" and "topological unitarity":

Obviously, if one exchanges the initial and dual Peano arithmetics, the "unitarity" (or "metric unitarity") and "topological unitarity" will be exchanged respectively, therefore "idempotently". Thus, "metrics" and "topology" at all rather than only "metric unitarity" and "topological unitarity" can be considered as idempotent to each other (or even as relative in a rigorous meaning forthcoming to be defined in (4) below). Metrics means implicitly an additional ordering due to "size" (understood very widely, i.e. as a synonym of any metric quantity) unlike topology considering the class of equivalence to that (or any) quantity ("size").

In essence, that idempotency is meant in set theory, but even as an equivalency, namely that in relation to a set "by itself", as a whole, and the same set (more precisely, postulated to be the same set), but as consisting of all its elements ${ }^{16}$. The corresponding problem in set theory due to the eventual identification of idempotency and set equivalency can be illustrated by the opposition between all natural numbers (all natural numbers are finite in virtue of the axiom of induction in Peano arithmetic) and the set of all natural numbers (that set is infinite in virtue of the axiom of infinity, e.g. or literally: in ZFC set theory). The interpretation as equivalence generates the well-known inconsistency between arithmetic and set theory in the foundation of mathematics (well-known or even famous by the Gödel (1931) incompleteness "theorems" inferring the dichotomy that arithmetics is either incomplete or inconsistent to set theory, both granted to be first-order logics to to the zero-order propositional logic).

On the contrary, the interpretation (as in the present pater) as idempotency is able to resolve that tension or inconsistency, but this is discussed in detail in other papers (Penchev 2020 August

\footnotetext{
${ }^{14}$ The utilization of concepts of Einstein's theories of relativity is neither metaphorical nor accidental. However, it is justified in other papers (Penchev 2020 October 20; 2021 June 8).

${ }^{15}$ The concepts of "Gödel mathematics" versus "Hilbert arithmetics" are formulated in other papers, and initially, in a paper in Bulgarian (2010).

${ }^{16}$ In fact, that duality of set, interpretable both idempotently and equivalently as above, generates a certain tension and even inconsistency in the foundation of mathematics rather than in set theory (Penchev 2020 July 24).
} 
2025; 2921 August 24). Analogically, metric and topological unitarities have not been identified but opposed as idempotent.

(2) The idempotence of "energy conservation" and "quantum-information conservation":

The idempotence of "metric unitarity" (what the standard use of "unitarity" is) and "topological unitarity" implies the idempotence of "energy conservation" and "quantum-information conservation". Maybe, a concise comment of the last statement is necessary to make the conclusion obvious:

A preliminary notice to it is that the tension between arithmetic and set theory in the foundations of mathematics (even interpretable by the dilemma of incompleteness versus inconsistency after Gödel) is now translated via the mathematical and physical bridge of the idempotence (i.e. interchangeability) of metric and topological unitarity (correspondingly) to the foundations of quantum mechanics (ant thus, to all physics), to which both conservations as well as their eventual idempotency belongs. Indeed, any metrics suggests a mapping in a relevant numerical set (e.g. and most often in physics, it is the rational numbers experimentally or the real or complex numbers theoretically), which can be always reduced to natural numbers, i.e. to arithmetic. On the contrary, topology does not need or even does not admit any metrics, or in other words, it means the class of equivalence of all possible metrics, but the concept of infinite set is crucially necessary for it, therefore being situated on the side of set theory once the opposition of it versus arithmetic has been meant. Even "metrics" is used to be interpreted as a synonym of 'finiteness' for it makes sense only to finite quantities (what are all measurable physical ones rather than only those in classical mechanics and physics).

A second preliminary notice consists in the observation that energy conservation needs the "size" of energy (due to which, "size" at all) and thus, metrics, therefore being naturally situated on the pole of arithmetics in the framework of the fundamental tension of mathematics. Accordingly, quantum-information conservation is to be situated oppositely, i.e. on the pole of set theory and infinity on the one hand, but on the other hand, it does not need "size", metrics at all, even not allowing for them, therefore granting to be similar physical objects so different in "size" and energy (but only if a relevant metrics is admitted) e.g. such as an electron and a star for both share wave functions as quantum entities in the final analysis.

Then, the idempotency of energy conservation and quantum-information conservation is to mean the idempotency of whether "size" (energy) is applicable or not. Meaning the relevant opposition in terms of "classical mechanics" (and classical physics as well) versus "quantum mechanics" (and quantum physics as well), one can exemplify the same relation as that of an internal observer ${ }^{17}$ (internal to the totality in the final, philosophical analysis, or respectively physically, to any wholeness, e,g, that postulated by quantum holism) versus an external observer (i.e. "out of the totality" or "out of the wholeness") as the latter is suggested in other papers (Penchev 2021 June 8; 2021 July 26).

\footnotetext{
${ }^{17}$ Any observer if the framework of both special and general relativity is granted without discussion to be an internal observer as far as the relevant metrics of physical quantities is involved necessarily.
} 
Furthermore, one can attach unambiguously an "external reference frame" to any external observer therefore suggesting a still more general principle of relativity than Einstein's one of "general relativity", and thus, involving a description of quantum mechanics in terms and concepts of general relativity, to which it occupies the area of a complementing theory referring to "external observer", respectively to the "still more general relativity" able to include all external reference frames as well (Penchev 2032 June 8).

The comment itself of the statement at issue above consists in an interpretation of Scolem's "relativity of the notion of set" (1922) fitted to the relation of metric unitarity (energy conservation) and topological unitarity (quantum-information conservation). The Norwegian scientist meant that the axiom of choice (as in virtue of the Löwenheim - Skolem theorem utilizing it, or directly) cancels the hierarchy of all infinities according their cardinal numbers and even the gap between "infinite set" and "finite set" if the latter is defined by means of the Dedekind set-theoretical finiteness ${ }^{18}$.

The last consideration can be directly related to the opposition of metrics and topology, and then exemplified by the concept of measurement in quantum mechanics generating a bijection between a well-ordering after measurement (being finite as by Peano arithmetic as by the Dedekind finiteness) and a relevant coherent state before measurement (being infinite and "topological"19 by itself). The application of the axiom of choice to the latter creates a second metrics (naturally exemplified by a well-ordering of all wave functions) and referable further to a statistical ensemble of measurements (being fundamentally random in any single case of measurement) obeying the rule that the characteristic function of the probability density distribution of that ensemble is the wave function at issue.

One can immediately notice that both metrics (meant in the last paragraph) can correspond directly to both anti-isometric (and furthermore, idempotent to each other) metrics of both dual separable complex Hilbert space utilized by quantum mechanics just for that idempotent anti-isometry. Thus can be visualized in an obvious way if one uses the definition of the dual Hilbert space by a relevant set of functionals (i.e. mappings so that their definition areas are also mappings which in turn can be considered as reverse functionals).

Continuing further the same visualization, one can attach energy conservation to the initial Hilbert space possessing the metrics of the field of all complex numbers (e.g. in virtue of the Born probabilistic interpretation of the square of the modulus) once it has been considered as the values of a functional. On the contrary, any metrics as to the dual Hilbert space is impossible (e.g. in virtue of the Heisenberg uncertainty), but nonetheless it can be involved secondarily, purely mathematically, due to the application of the axiom of choice to all wave functions. The eventual law of quantum-information conservation means that only hypothetical metrics

\footnotetext{
${ }^{18}$ That is: by the absence of any constantly acting bijection between an infinite set and a Dedekind finite set.

${ }^{19}$ The quotation marks are due to the following: of course, any set is "topological" in the sense that at least topology can be attached to it. However, one means here that topology is able to correspond to "topological unitarity".
} 
"caused" by the axiom of choice applied to the separable complex Hilbert space of all possible wave functions.

Then, the mutual idempotence of energy and quantum-information conservation seems to be already obvious just because of the idempotency of the dual Hilbert spaces. One is to add the purely hypothetical and only mathematically existing metrics in virtue of the axiom of choice meant in quantum information conservation, which is verified experimentally by all phenomena of entanglement. Accordingly, that metrics refers practically to probability density distributions and their interactions (particularly, able to violate Bell's inequalities or many others derivative or similar to them).

So, quantum-information conservation means, loosely speaking, the conservation of possibility (measurable as probability and groupable into probability density distributions, the characteristic functions of which are the wave functions meant literally in quantum-information conservation), and energy conservation, the conservation of actuality. However, the former conservation is able to describe quantitatively also their mutual transformations, and therefore, how energy can appear from nothing (accumulating enough possibilities as actuality) or vice versa: vanish into nothing (reversely transforming actuality into possibilities) and particularly debunking the myth of the "Big Bang" relying only on our prejudice of the "omnipresent" energy conservation in the final analysis.

(3) About the relativity of "energy conservation" and "quantum-information conservation":

The concept of "idempotence" (being commonly accepted to dual spaces such as dual Hilbert space) has been introduced and utilized until now. Meanwhile, it has been linked to Skolem's "relativity of the notion of set", though. Furthermore, the latter seems to be more relevant to ideas promoted in the present section, namely speaking about information at all, therefore erasing distinction (meant in the previous section) between the "finite", classical information and the "infinite" quantum information.

Following Skolem's approach once the axiom of choice (being equivalent to the well-ordering in the framework of the standard, e.g. ZFC set theory meant here) has been involved implicitly by the relation of choice and ordering, the relativity of finiteness and infinity also to information ${ }^{20}$ (i.e. the unity or generalized invariance of both classical and quantum information) is a natural conjecture, even almost obvious:

Indeed, infinity can be understood by virtue of the concept of "infinite set" as: (1) an observation "outside" to all natural numbers being finite "inside", due to the axiom of induction; (2) the concept of "infinite" set can be postulated to be: (2.1) the internal structure of any arithmetical unit therefore promoting the approach of Hilbert arithmetic by that and (2.2) establishing a perfect symmetry (borrowed from the approach of the Standard model in physics) of "infinite set" being both ultimate framework of "all" and initial element of anything situated between those two extremes.

${ }^{20}$ The present paper uses "information" as a synonym (according to common use) of the proper, i.e. finite. "classical" information as "information at all" (both "classical" and quantum). This would not generate any mess as far as which use is meant is unambiguously determined by the corresponding context. 
Then, the concept of information (already independently of being infinite, quantum or finite, classical) is necessary to deliver that duality inherited by Hilbert space for its definitive duality. Indeed, the unit of classical information, a bit, means just the choice of two equally probable alternatives, which now are only interpreted as dual to each other. In turn, the init of classical information, a qubit, means the choice of an infinite set of alternatives, which now are only interpreted as all units of Hilbert arithmetic, on the one hand, and the internal structure of each of those units, on the other hand. The probability density distribution regulates the relation (i.e. the "bijection" of an infinite set into a "variable" finite set and avoiding any eventual inconsistency in virtue of the Dedekind finiteness) of the two idempotent levels just as relative to each other:

This interrupts the established representation of common sense that infinity is more than finiteness complementing by the opposite viewpoint, according to which (and not less than the former) infinity is less than finiteness. The contradiction is reconciled by the idempotent duality of the former and latter viewpoints.

Skolem introduced "relativity of set" in the considered context being perhaps influenced by the triumph of Einstein's relativity at that time (the twenties of the 20th century). Nonetheless, the use of relativity transferring it from a fundamental physical theory to a mathematical one is exceptionally fruitful to the approach here. Anyway, a difference between the two uses is to be emphasized:

Einstein's relativity is quantitative, metrical and means the (metric) invariance of one or other quantity. On the contrary, Skolem's relativity is qualitative, topological and means the (topological) invariance of one or other quality (such as "infinity" in "infinite set" and "finiteness" in "finite set", literally). However, one can immediately notice that the approach of the present paper (for example by the relativity of the conservation of whether energy or quantum information) erases that distinction seeming to be insurmountable at first glance.

So, one may speak of a single unitarity (which remains a single one in classical mechanics and reflectable by energy conservation), however doubled necessarily as to any dual space (particularly, to both dual spaces meant by quantum mechanics and belonging to the same separable complex Hilbert space): the one, proper or initial dual space conserves the energy conservation of classical mechanics ${ }^{21}$; the other one appears necessarily once its dual space has been involved (as quantum mechanics was forced to do) therefore generalizing metric unitarity by topological unitarity and implying quantum-information conservation (speaking loosely, independent of "size" and allowing to be compared micro-, macro-, and mega- physical objects).

Another result because of the introduction and interpretation of dual space (along with quantum information, but closely linked to it) is information whether quantum, infinite, or "classical", finite to become a fundamental concept in the foundation of both mathematics and physics, furthermore allowing for their merging in a Pythagorean manner drastically

${ }^{21}$ This allows one to speak of the "conservation of energy conservation in quantum mechanics" (Penchev 2020 October 5). However, it is to be necessarily complemented by quantum-information conservation due to the availability of dual space, which is absent fundamentally from the mathematical formalism of classical mechanics. 
contradicting both Cartesian dualism and episteme of Modernity, in which physics and mathematics are gapped by the abyss of "body" versus "mind" correspondingly.

Indeed, classical information is able to represent the relation of the dual counterparts; and quantum information, that of element to the space, to which it belongs. Then, the unification of information whether classical or quantum means both dual spaces as a whole (eg. as Hilbert space). In other words, information is unavoidably to be involved in the foundation if the separable complex Hilbert space (due to its dual structure) has been established as the basic mathematical formalism of quantum mechanics.

In the final analysis, information represents quantitatively the relation of "choice" and "ordering" in both physics and mathematics: that relation in set theory is postulated by the axiom of choice and its provable equivalence to the well-ordering "theorem".

\section{CHOICE AND ORDERING IN PHILOSOPHY; TRANSCENDENTALITY} ORIGINATING FROM THE TOTALITY

The relation of choice and ordering (and thus implicitly, the concept of information) can be discovered in Western philosophy (which is natural if it is considered to be that science, the subject of which are foundations at all) after it can be revealed in the foundations of both mathematics and physics and even appeared before the latter:

Indeed, Cartesian dualism divided radically the Self from the world established some centuries earlier and as a general philosophical problem how they would be unified by themselves (i.e. without "God's help") anyway. Kant's "Copernican revolution" of transcendentalism at least as a fundamental principle underlying following Western philosophical doctrines seems to be the single solution even until now. Previous papers (e.g.: Penchev 2021 April 12; 2021 july 26) demonstrate that it can be modified and thus verified as the falsifiable "scientific transcendentalism", in turn discoverable implicitly in the foundations of both physics and mathematics, furthermore to unify them not only between each other, but with philosophy as well.

The present paper following the same general direction clears up how that scientific transcendentalism can be interpreted by "choice", "ordering", and "information" (as their relation) even philosophically therefore revealing those concepts as "categories" able to unify physics, mathematics, and philosophy. The approach of their philosophical interpretations needs "the totality" borrowed from philosophical transcendentalism but revealable in physics and mathematics, e.g. as "wholeness", "completeness", "ensemble", system, etc. in conceptions as "quantum holism", the "self-foundation of mathematics", the "completeness of quantum mechanics", etc.

According to the pathway from transforming philosophical transcendentalism into a formal hypothesis to be applicable to mathematical or physical research, and exhibited in detail in other papers (Penchev 2020 October 20; 2021 July 26), the totality is defined uniquely as containing its externality within itself unlike any other entity. Now, externality and internally will be thought to be mutually exchangeable, or mathematically, idempotent or dual to each other. That property seems to be naturally inferable from the definition of the totality. Furthermore, "choice" can be 
attached only to one of them (intuitively preferable is that to be the externality of the totality, but this not formally necessary); then, "ordering" is to be linked to the corresponding internality. In other words, "externality" is thought as the extension of the property of choice, and internality, of ordering; or respectively: vice versa.

Then, their relation, "information" will regulate the mutual transformations or transitions between them, e.g. so as the axiom of choice being equivalent the well-ordering theorem manages "choice" and "ordering" in the framework of set theory. Though, unitarity is initially defined to vector spaces such as Hilbert spaces, it can be generalized to the concept of "set" (granted to be the most fundamental structure in mathematics if "set" underlies it) relying only on the axiom of choice: unitarity means just the equivalence of "choice" and "ordering" in virtue of the equivalence if the axiom of choice and the well-ordering "theorem".

The transition between "choice" and "ordering" is necessarily discrete for being a transition between two different qualities: then, a bit of information is to be defined just the minimal discreteness of that discrete transition ${ }^{22}$, in other words, as a unit of it and thus, isomorphic to the usual unit of arithmetic therefore being able to obey Peano axioms. However and unlike the arithmetical unit, a bit possesses an internal structure and due to which it can be distinguished from a qubit. This implies the necessity for "arithmetical unit" to be considered as classes of equivalence of bits or qubits (e.g. as in Hilbert arithmetic).

Then, if information be postulated as it is defined above, the totality exists in two forms, eventually equivalent to each others, if unitarity holds: as an amorphic (or "coherent" in quantum mechanics) state as a well-ordered (or "temporal" in both classical and quantum physics) state. Thus, unitarity can be interpreted even philosophically rather than only set-theoretically.

Unitarity applied to quantum mechanics implies energy conservation, and applied in the framework of quantum phenomenology implies the analogical or mathematically isomorphic conservation of the Self. Speaking ontologically, one can even identify unitarity and conservation. Anyway, one can distinguish unitarity from the being as a philosophical category, e.g. as "Zeit" and "Sein" correspondingly (following Heidegger) therefore admitting the option of non-temporal Being, in the scope of which quantum information can be thought though rather as a specific scientific or only physical notion partly corresponding to the philosophical category, e.g. as it is allowed above.

Then, the law of quantum-information conservation not requiring unitarity unlike energy conservation or any temporality can be reflected even ontologically, meaning the mutual transformation of possibility (probability, mathematically) and actuality both interpreted as forms of Being conserving itself by itself (and unlike Time).

\footnotetext{
${ }^{22}$ Indeed, that minimal discrete transition of a bit means an elementary and thus minimal ordering, namely that of two elements (for the ordering of a single element does not make sense) therefore consisting of a single choice of which element to be first (respectively, second), and the other element occupies the other position necessarily.
} 


\section{INSTEAD OF CONCLUSION: AGAIN AND AGAIN ABOUT THE SHARED FOUNDATION OF PHYSICS, MATHEMATICS AND PHILOSOPHY}

The concepts of "choice" and "ordering" as well as their relation by "information" and "unitarity" is considered in three areas: physics, mathematics and philosophy. As in other papers, the intention is to demonstrate the option of their common basis, the shared and uniform methods of research and structure, which can be interpreted equally well as physical, mathematical and/ or philosophical.

That approach contradicts the standard organization of scientific cognition established still since Descartes's edge, after which physics is opposed and even gapped to or from mathematics therefore needing philosophy to mediate between them by the "figure of human being" in turn established to be the fundamental subject of Western philosophy since Socrates.

Michel Foucault in Les mots et les choses ("Words and Things" localized on the two opposed shores of that abyss and therefore needing "human being" to mediate between them) suggested the hypothesis, though, that the figure of human being situated according to the organization in knowledge (or Foucault's "episteme") will vanish since it is possible only in that historically determined episteme. This would crucially influence how philosophy realizes itself (i.e. as a humanitarian science until now), however forced already to abandon "human being" for a future episteme, in which the constellation of any possible cognition would be radically different or even not needing that concept at all. The present investigation discusses an admissible epistemic change cancelling that abyss of Cartesian dualism and thus the figure of human being "would be erased, like a face drawn in sand at the edge of the sea" just as in Foucault (1966)'s metaphor and sharing his idea about a forthcoming change in human cognition.

Indeed, if physics and mathematics can be unified (moreover if the unification can be identified as philosophy), the contradictory finite-infinite space fetching between its two extremes by cognizing human being no longer exists because of the coincidence of physics and mathematics and this would correspond to the pre-Socratic epoch in philosophy, more exactly to Pythagoreanism though in a contemporary, "quantum" form. The long run in the history of philosophy, begun still for Socrates's "human revolution" in it and reconfirmed by Descatrtes as to Modernity, has ended ... 


\section{REFERENCES:}

Born, M. (1954) "Max Born - Nobel Lecture: The Statistical Interpretations of Quantum Mechanics," Nobelprize.org. Nobel Media AB 2014. Web. (13 Jul 2016.)

http://www.nobelprize.org/nobel prizes/physics/laureates/1954/born-lecture.html

Dedekind, R (1888) Was sind und was sollen die Zahlen? Stetigkeit und Irrationale Zahlen (Herausgeber: Stefan Müller-Stach), Berlin, Springer Spektrum, 2017.

DeWitt, B. S., Graham, N., eds. (1973) The many-worlds interpretation of quantum mechanics: a fundamental exposition. Princeton, NJ: University Press.

Foucault, M. (1966) Les mots et les choses. Paris: Gallimard.

Einstein, A., Podolsky, B., Rosen, N. (1935) "Can Quantum-Mechanical Description of Physical Reality Be Considered Complete?" Physical Review 47 (10): 777-780.

Everett III, H. (1957) “'Relative State' Formulation of Quantum Mechanics," Reviews of Modern Physics 29 (3): 454-462.

"Institute for Advanced Study, Princeton, NJ, Univalent Foundations Program" (2013) Homotopy type theory: univalent foundations of mathematics. Princeton, NJ: Lulu Press, Univalent Foundations Program.

Kochen, S., Specker, E. (1968) “The Problem of Hidden Variables in Quantum Mechanics," Journal of Mathematics and Mechanics 17 (1): 59-87.

von Neumann, J. (1932) Mathematische Grundlagen der Quantenmechanik. Berlin: Springer, pp. 167-173.

Penchev, V. (2021 August 24) "Hilbert arithmetic as a Pythagorean arithmetic: arithmetic as transcendental," SSRN, https://papers.ssrn.com/sol3/papers.cfm?abstract id=3909610 or https://dx.doi.org/10.2139/ssrn.3909610 .

Penchev, V. (2021 July 26) "Quantum phenomenology as a "rigorous science": the triad of epoché and the symmetries of information," SSRN, https://papers.ssrn.com/sol3/papers.cfm?abstract id=3892039 or https://dx.doi.org/10.2139/ssrn.3892039 .

Penchev, V. (2021 June 8) "The Symmetries of Quantum and Classical Information. The Resurrected 'Ether' of Quantum Information," SSRN, https://papers.ssrn.com/sol3/papers.cfm?abstract id=3861105 or https://dx.doi.org/10.2139/ssrn.3861105.

Penchev, V. (2021 April 12) "Both Classical \& Quantum Information; Both Bit \& Qubit: Transcendental Time. Both Physical \& Transcendental Time," SSRN, https://dx.doi.org/10.2139/ssrn.3823665 , https://papers.ssrn.com/sol3/papers.cfm?abstract id=3823665 .

Penchev, V. (2020 October 20) "Two deductions: (1) from the totality to quantum information conservation; (2) from the latter to dark matter and dark energy," SSRN, https://dx.doi.org/10.2139/ssrn.3683658, https://papers.ssrn.com/sol3/papers.cfm?abstract id=3683658 .

Penchev, V. (2020 October 5) "Quantum-Information Conservation. The Problem About 'Hidden Variables', or the 'Conservation of Energy Conservation' in Quantum Mechanics: A Historical Lesson for Future Discoveries," SSRN, https://papers.ssrn.com/sol3/papers.cfm?abstract id=3675319 or https://dx.doi.org/10.2139/ssrn.3675319.

Penchev, V. (2020 August 25) "The Relationship of Arithmetic As Two Twin Peano Arithmetic(s) and Set Theory: A New Glance From the Theory of Information," SSRN, https://papers.ssrn.com/sol3/papers.cfm?abstract id=3656179, https://dx.doi.org/10.2139/ssrn.3656179 .

Penchev, V. (2020 August 5) “A Class of Examples Demonstrating That 'P $\neq$ NP' in the 'P Vs NP' Problem," SSRN, https://papers.ssrn.com/sol3/papers.cfm?abstract id $=3647038$ or https://dx.doi.org/10.2139/ssrn.3647038. 
Penchev, V. (2020 July 24) “Skolem's 'Paradox' As Logic of Ground: The Mutual Foundation of Both Proper and Improper Interpretations," SSRN, https://dx.doi.org/10.2139/ssrn.3640109 or https://papers.ssrn.com/sol3/papers.cfm?abstract id=3640109.

Penchev, V. (2020 July 22) "A Mathematical Model of Quantum Computer by Both Arithmetic and Set Theory," SSRN, https://papers.ssrn.com/sol3/papers.cfm?abstract id=3638937 or https://dx.doi.org/10.2139/ssrn.3638937.

Penchev, V. (2019) "From the Principle of Least Action to the Conservation of Quantum Information in Chemistry: Can One Generalize the Periodic Table?" Chemistry: Bulgarian Journal of Science Education 28 (4): $525-539$ (in SSRN: https://papers.ssrn.com/sol3/papers.cfm?abstract id=3644084).

Penchev, V. (2010) "Insolvability of the first incompleteness theorem. Gödel and Hilbert mathematics," Philosophical Alternatives 19 (5): 104-119 (in Bulgarian: "Неразрешимост на първата теорема за непълнотата. Гьоделова и Хилбертова математика”).

Perelman, G. (2002) "The entropy formula for the Ricci flow and its geometric applications," arXiv: https://arxiv.org/abs/math/0211159.

Perelman, G. (2003) "Ricci flow with surgery on three-manifolds," arXiv: https://arxiv.org/abs/math/0303109.

Perelman, G. (2003) "Finite extinction time for the solutions to the Ricci flow on certain three-manifolds," arXiv: https://arxiv.org/abs/math/0307245.

Poincaré, M. H. (1904) "Cinquième complément à l'Analysis situs," Rendiconti del Circolo Matematico di Palermo 18 (1): 45-110. https://www.maths.ed.ac.uk/ v1ranick/papers/poincare2009.pdf.

Skolem, T. (1922) "Einige Bemerkungen zur axiomatischen Begründung der Mengenlehre," in Matematikerkongressen $i$ Helsingrofs den 4-7 Juli 1922, Den femte skandinaviska matematikerkongressen, Redogörelse. Helsinki: Akademiska-Bokhandeln, 1923, pp. 217 - 232.

Wheeler, J. A., Zurek, W. H., eds. (1983) Quantum theory and measurement. Princeton, N.J.: University Press. 